\title{
Clima organizacional en las instituciones de salud desde una perspectiva teórica
}

\author{
Graciela Maribel Fajardo Aguilar \\ Universidad Técnica de Machala, Ecuador \\ gfajardo@utmachala.edu.ec \\ https://orcid.org/0000-0003-1152-6414 \\ Verónica Jacqueline Almache Delgado \\ Universidad Técnica de Machala, Ecuador \\ vjalmache@utmachala.edu.ec \\ https://orcid.org/0000-0001-5403-1863 \\ Heydi Katherine Olaya Santos \\ Dirección Distrital 07D02-Machala-Salud, Ecuador \\ heydi_290494@hotmail.com \\ https://orcid.org/0000-0002-2578-2438
}

\section{Resumen}

En el sector de la salud cada institución es única y todo grupo humano desarrolla características especiales, así pues en la actualidad, estudiar el clima organizacional dentro del ámbito de la salud es una de las herramientas estratégicas que ayudan al mejoramiento continuo de la institución, dado que, mediante su análisis se pueden establecer factores clave que permiten incrementar la calidad de vida laboral de los distintos profesionales de la salud, y con ello la calidad de los servicios médicos que se ofrecen a la población. A partir de ello, el objetivo del presente manuscrito se centra en realizar una recopilación bibliográfica con la finalidad de presentar el origen y la evolución del término clima organizacional enfocado a instituciones de salud y cuyos actores principales son los distintos profesionales que interactúan en este sector. Para ello, la metodología empleada está dada bajo un diseño hermenéutico, apoyada por una investigación bibliográfica con el fin de recopilar información 
de diferentes fuentes secundarias y plasmar información útil que servirá como referencia para otras investigaciones. Bajo estos antecedentes y como principales hallazgos se pudo determinar que el conocimiento del clima organizacional en las distintas entidades de salud suministra retroinformación en relación a los procesos que determinan los comportamientos institucionales, permitiendo también introducir cambios planificados tanto en las conductas y actitudes de cada uno de los miembros implicados.

Palabras clave: Calidad de vida laboral, clima organizacional, herramientas estratégicas, profesionales de la salud, servicios médicos.

\begin{abstract}
In the health sector, each institution is unique and every human group develops special characteristics, so at present, studying the organizational climate within the field of health is one of the strategic tools that help the continuous improvement of the institution, given that, through its analysis, key factors can be established that allow increasing the quality of work life of the different health professionals, and with it the quality of the medical services offered to the population. From this, the objective of this manuscript is focused on making a bibliographic compilation with the purpose of presenting the origin and evolution of the term organizational climate focused on health institutions and whose main actors are the different professionals that interact in this sector. To do this, the methodology used is given under a qualitative approach, supported by a bibliographic investigation in order to gather information from different secondary sources and capture useful information that will serve as a reference for other investigations. Under these antecedents and as main findings it was possible to determine that the knowledge of the organizational climate in the different health entities provides feedback in relation to the processes that determine the institutional behaviors, allowing also to introduce planned changes in both the behaviors and attitudes of each of the members involved.
\end{abstract}

Keywords: Quality of work life, organizational climate, strategic tools, health professionals, medical services. 


\section{Introducción}

En los actuales entornos competitivos por los cuales atraviesa la atención médica en general, se reconoce la importancia de la fuerza laboral para el desarrollo de sistemas de salud y la necesidad de conseguir un clima laboral positivo es cada vez más importante para la satisfacción, desempeño y productividad de los profesionales de la salud en general (Munyewende, Rispel y Chirwa, 2014). De esta manera, un clima organizacional positivo incrementa de forma significativa el compromiso de los distintos profesionales con su trabajo y ayuda también a la reducción de la intención de abandonar su lugar de trabajo (Mohamadzadeh, Ashgholi, Rafii y Bahrani, 2015). Por lo tanto, establecer un clima laboral positivo que influya de forma directa en el comportamiento, la moral y la actitud del personal de salud es una de las principales preocupaciones para los distintos proveedores de atención médica (Pinpin, Cheung y Meiche, 2012).

Bajo este contexto, al revisar la evolución del concepto del clima organizacional, se observa que sus primeras definiciones se remontan a la década de los años treinta, donde se lo planteaba como el comportamiento humano en función del ambiente de cada individuo (Pedraza y Bernal, 2018). No obstante, recién se empieza a discutir en la literatura a finales de los años sesenta para comprender el comportamiento relacionado con el trabajo de los empleados (Berberoglu, 2018). En la década de los años ochenta se inicia con el planteamiento de que el clima organizacional es una cualidad relativamente duradera del ambiente interno de la organización; es decir, se lo empieza a estudiar como un conjunto de propiedades o características percibidas en el entorno de trabajo (Gruesso, 2016); sin embargo, a pesar de las acotaciones realizadas, es un fenómeno que se mantiene aún vigente en la agenda de investigación, estimando prevaleciente su estudio por las implicaciones que su gestión constituye en el comportamiento colectivo de una institución, dado que influye directamente en la motivación de sus trabajadores y en el desempeño del trabajo realizado (Pedraza y Bernal, 2018).

A partir de ello, el clima organizacional puede ser definido como los patrones recurrentes de actitudes, comportamientos y sentimientos que definen la vida en una institución y que están más relacionados con el ambiente y los valores; es decir, donde y con los cuales interactúan las personas (Ramírez y Domínguez, 2012). Asimismo, puede ser considerado como un conjunto de características que describen una empresa u organización y que la diferencian de otras (Gan y Berbel, 2007).

En la actualidad, estudiar el clima organizacional dentro del ámbito de la salud es una de las herramientas estratégicas que ayudan al mejoramiento continuo de la institución, dado que, mediante su análisis se pueden establecer factores clave que permiten incrementar la calidad de vida laboral de los distintos profesionales de la salud, y con ello la calidad de los servicios médicos que se ofrecen a la población, esto debido a la influencia que este fenómeno mantiene sobre el desempeño y la motivación de los trabajadores, lo cual trae consigo importantes consecuencias en la productividad, compromiso, satisfacción y calidad del trabajo. Por lo tanto, derivado de estas aseveraciones se puede señalar que el clima organizacional representa la antesala en el aseguramiento de la calidad de los servicios de salud (Bernal, Pedraza y Sánchez, 2015). 
Con estos antecedentes, el objetivo del presente manuscrito se centra en realizar una recopilación bibliográfica con la finalidad de presentar el origen y la evolución del término clima organizacional enfocado a instituciones de salud y cuyos actores principales son los distintos profesionales que interactúan en este sector (administrativos, médicos, enfermeros, auxiliares de enfermería, etc.). Para ello, la metodología empleada está dada bajo un enfoque cualitativo, apoyada por una investigación bibliográfica con el fin de recopilar información de diferentes fuentes secundarias y plasmar información útil que servirá como referencia para otras investigaciones.

\section{Marco Teórico}

\section{Origen y Evolución}

El clima organizacional nace de la idea de que el hombre vive en ambientes complejos y dinámicos, dado que las instituciones se encuentran conformadas por personas, colectividades y grupos que generan distintos comportamientos y que afectan de forma directa dicho ambiente. En este sentido, el origen del clima organizacional es la sociología; en donde el concepto de organización dentro de la teoría de las relaciones humanas enfatiza la importancia del hombre en su función del trabajo y por su participación en un sistema social (García, 2009).

El concepto de clima organizacional se originó a fines de la década de 1950 cuando los científicos sociales estudiaron las variaciones en los entornos de trabajo. El concepto se hizo popular en la literatura industrial y organizacional, de manera particular en los años 1960 y 1970 con el libro de Litwin y Stringer (Githinji y Gachunga, 2017). No obstante, en la actualidad existe una serie de investigaciones que vinculan el clima organizacional con varios factores de resultado organizacionales e individuales importantes. Por ejemplo, distintos estudios han demostrado una relación directa entre el clima organizacional y los factores de producción, como el bienestar y la salud de los empleados, la satisfacción laboral, el compromiso organizacional, la rotación y las intenciones de rotación (Bronkhorst, Tummers, Steijn y Vijverberg, 2015).

De los distintos enfoques que abordan la conceptualización del clima organizacional, aquel que ha demostrado mayor utilidad es el que utiliza como componente indispensable las percepciones que el trabajador tiene de los procesos y estructuras que ocurren en un contexto laboral, de ahí que la calidad del ambiente juega un papel preponderante en la percepción sobre el clima de una organización, no obstante, estas percepciones dependen en gran medida de las interacciones, actividades y experiencias que cada miembro tenga con la institución: por consiguiente se refleja la interacción entre las características organizacionales y personales (Segredo y Reyes, 2015).

Cuando se hace alusión al clima organizacional se hace mención a la atmosfera interna en que funciona la institución, la misma que se encuentra influenciado de forma lógica por la calidad de sus relaciones con el contexto en el cual se recibe y envía la información para la toma de decisiones. Finalmente, en la práctica misma, dentro del clima influyen todos los factores subjetivos y objetivos presentes en las instituciones, los estilos de dirección, las formas y condiciones de trabajo, las relaciones interpersonales, los sistemas de compensación, los procesos comunicacionales pudiendo ser estos externos e internos, ascendentes y descendentes, 
formales e informales (De La Cruz y Huaman, 2016).

\section{Clima organizacional}

El clima organizacional constituye la percepción compartida que el capital humano de una institución se forma sobre su ambiente de trabajo pudiendo ser formal e informal. Asimismo, representa fundamentalmente el significado que los trabajadores otorgan a sus experiencias de su ambiente laboral, se considera en esa valoración las políticas y rutinas organizativas (prácticas y procesos), así como las conductas que son recompensadas y reconocidas en el contexto organizacional, las cuales influyen en las actitudes de los empleados permitiendo esto la adaptación del comportamiento individual a las necesidades y exigencias de la organización en función del significado que dan a sus experiencias laborales (Pedraza y Bernal, 2018).

En este sentido, el clima organizacional representa un concepto que habitualmente se ha establecido y definido como un complejo constructo, que constituye un conjunto diverso de características del contexto laboral, las mismas que son percibidas por los trabajadores y la importancia que dan ellos a estos aspectos organizacionales son los que influyen de forma directa en su comportamiento dentro de la institución (Iglesias y Sánchez, 2015). Es por esta razón, la importancia de realizar investigaciones que aporte más evidencia empírica sobre el estado del arte de este fenómeno organizacional.

Por otro lado, dentro del contexto de la salud, el personal involucrado debido a la dificultad de su trabajo requiere un clima laboral adecuado que les brinde una sensación de valor cuando realizan su trabajo, dado que varios estudios han identificado una serie de factores que ayudan a la estructuración de un clima organizacional favorable para un mejor desempeño de los profesionales de la salud entre los cuales se pueden destacar: trabajo en equipo, preocupación por las personas, facilitación del trabajo, comunicación entre pares, participación en la toma de decisiones, relación usuario/médico, servicio al cliente externo y compensación (Guevara, 2018; Bonilla, Macero y Mora, 2018).

De esta manera, los entornos laborales poco saludables son un determinante importante de varios resultados asociados con el trabajo, en el cual se pueden incluir aspectos tales como la insatisfacción laboral y el agotamiento profesional (Pérez, 2014). Mientras que, los profesionales que perciben sus entornos de trabajo como amigables y solidarios presentan mayores probabilidades de exhibir un buen desempeño y comportamientos positivos con sus pacientes y entre pares. A esta última acotación, es importante mencionar que un clima organizacional adecuado conduce a la innovación e inspiración en la institución y juega un papel preponderante en la consecución o no de los objetivos institucionales planteados (Yataco, 2014).

Así pues, el clima laboral organizacional se encuentra asociado con distintos tipos de desempeño organizacional, especialmente el desempeño de los profesionales de la salud. Dentro del entorno hospitalario, el desempeño de éstos se reconoce como un componente integral en la provisión de atención médica de calidad y desempeña un papel clave en las decisiones del personal, como la promoción y la retención. En tal virtud, el rendimiento constituye el proceso de evaluar qué tan bien los médicos, enfermeras, auxiliares de enfermería, entre otros, realizan su trabajo en comparación 
con un conjunto de estándares preestablecidos por la institución (Contreras, Reynaldos y Cardona, 2015).

Bajo este contexto, el desempeño de los distintos profesionales de la salud se fundamenta en qué tan bien realizan su trabajo en relación con los estándares previamente establecidos. Además, representa la posibilidad de desarrollar actividades particulares para lograr con éxito los objetivos establecidos dentro del marco del tiempo dado y las limitaciones de las partes interesadas, en otras palabras, el término desempeño hace mención a la eficacia con que un profesional de la salud lleva a cabo los roles y responsabilidades laborales que le son encomendadas (Forrellat, 2014).

El desempeño de cada uno de los profesionales involucrados en el sector de la salud es esencial para conseguir las metas y objetivos de una institución, dado que la atención al paciente constituye el núcleo del desempeño en las organizaciones sanitarias, pues los pacientes quieren ser tratados bien, saber que éstos profesionales se encuentran bien informados, capacitados y competentes, tener una alta calidad en todo instante y sobre todo quieren que dentro de la interacción que se genera exista una actitud humana y afectuosa, que los haga sentir cómodos y seguros (Artaza et al., 2016).

En tal virtud, la competencia tanto de médicos, enfermeras y auxiliares se puede mejorar a través del análisis del desempeño y la identificación de las necesidades de capacitación para mejorar el servicio que se está prestando. Asimismo, la medición frecuente del desempeño laboral crea un sistema de atención médica que brinda atención eficiente, efectiva y de alta calidad (Romero, Souto y García, 2018).

Diferentes estudios revelan que, para mejorar el desempeño de las distintas personas que se encuentran inmersas en la actividad de la salud, los administradores de los hospitales e instituciones sanitarias en general deben crear un ambiente que valore a los profesionales, los empodere de una forma que puedan lograr el potencial latente y puedan fortalecer o cambiar de forma positiva la cultura de la institución (Montoya y Boyero, 2016).

\section{Factores que influyen en el clima organizacional}

Bajo la perspectiva de Santana (2018) se han identificado nueve factores que influyen directamente para el establecimiento de un adecuado clima organizacional. A partir de ello, a continuación, dentro de la figura 1, se describen dichos factores. 


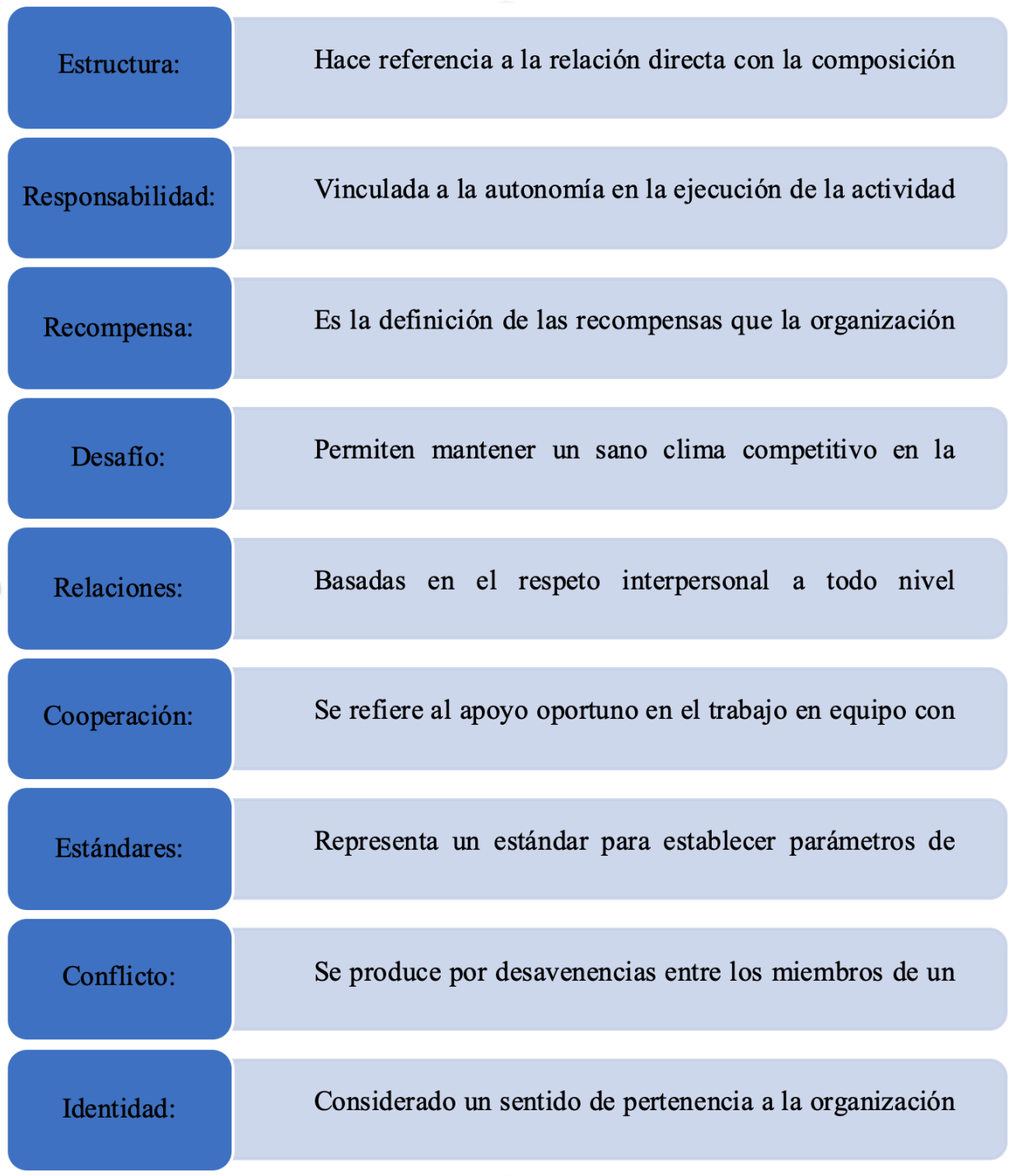

Figura 1. Factores que influyen en el clima laboral

Fuente: A partir de Santana, M. (2018)

En este sentido, cada uno de estos factores generan distintos tipos de percepción que influyen de forma directa en la moral laboral individual y la suma de todos estos componentes da origen al clima organizacional.

\section{Metodología}

El presente manuscrito se encuentra dado bajo un diseño fenomenológico hermenéutico que según Hernández y Mendoza (2018) constituye en la interpretación de la experiencia humana y los textos de la vida, no sigue reglas específicas, pero considera que es producto de la interacción dinámica entre las siguientes actividades: a) concebir un fenómeno o problema de investigación, b) estudiarlo y reflexionar sobre este, c) descubrir categorías y temas esenciales del fenómeno, d) 
describirlo y e) analizarlo. Bajo este contexto, la investigación bibliográfica desarrollada se centró en la revisión de literatura de diferentes fuentes de información de tipo secundarias, que engloba libros, investigaciones realizadas con anterioridad y artículos científicos publicados en diferentes revistas a nivel nacional e internacional, buscando que las publicaciones sean las adecuadas sin restricción de idioma o año de publicación. A partir de ello, a continuación, dentro de la tabla 1, se describen las principales bases científicas y la cantidad de artículos que fueron necesarios para la estructuración del estudio en cuestión.

Tabla 1.

Bases de datos analizadas

\begin{tabular}{llc}
\hline \multicolumn{1}{c}{ Descripción } & \multicolumn{1}{c}{ Base científica } & \# de ejemplares empleados \\
\hline \multirow{3}{*}{ Artículos científicos } & Latindex & 4 \\
& Scielo & 7 \\
& Scopus & 8 \\
\hline Libros & & 2 \\
\hline Proyectos de investigación & 5 \\
\hline Informes & & 1 \\
\hline Total & & $\mathbf{2 7}$ \\
\hline
\end{tabular}

Elaboración propia

\section{Resultados}

A partir de lo expuesto en el epígrafe anterior, seguidamente se presentan los principales hallazgos en torno a dichos documentos analizados. De forma general, se pudo obtener información de 27 fuentes confiables y con una calidad de información muy buena para procesar la información a través de un proceso de lectura comprensiva y un posterior análisis por parte de los investigadores. Tal como se observa en la tabla 1, la información proviene de distintas fuentes bibliográficas de las cuales se detalló tanto categorías y subcategorías implícitas en el clima organizacional dentro de las instituciones de salud que fueron utilizados en su debido tiempo en cada una de las investigaciones tomadas en consideración.

En este sentido, la revisión de todos estos documentos muestra la importancia del clima organizacional dentro del contexto de la salud; es por ello que, para tener un horizonte más amplio, se han establecido percepciones donde los profesionales de la salud requieren de un clima laboral adecuado que les brinde una sensación de valor. A partir de ello, López, Casique y Díaz (2014) identifican ciertos factores que contribuyen a un clima favorable e indirectamente afectan de forma positiva el desempeño de los profesionales involucrados, los cuales incluyen por ejemplo la facilitación del trabajo, preocupación por las personas, trabajo en equipo, toma de decisiones, participación, comunicaciones, servicio al cliente, calidad, relación personal/médico y compensación. Cada uno de los factores citados ayudan a la construcción de un ambiente agradable de trabajo mejorando de esta forma el desempeño laboral y la calidad del servicio.

Asimismo, los estudios sobre el clima laboral permiten recabar información sobre las percepciones 
que en un momento dado tienen los trabajadores con relación a ciertos factores de la organización, mismos que a su vez influyen de forma positiva o negativa en el desempeño de los trabajadores y de la organización. En tal virtud, un estudio realizado por Dos Santos y Guirardello (2016) determinó la relación entre el entorno laboral, la seguridad del paciente y la calidad de la atención en un hospital. El estudio además concluyó que el ambiente de práctica profesional en las instituciones de salud impacta en la calidad y la seguridad de la atención brindada a los pacientes.

En este mismo contexto, un estudio realizado en Egipto tuvo como objetivo examinar la relación entre el clima organizacional y los resultados laborales de las enfermeras, y encontró que el clima organizacional se correlacionó con el resultado laboral de las enfermeras (Hassan y Gaber, 2015). En este sentido, de acuerdo con la literatura recabada, el clima organizacional se correlaciona con la efectividad del trabajo, el resultado del trabajo y el desempeño organizacional que se considera un parámetro importante en la profesión de la salud.

\section{Conclusiones}

El presente manuscrito fue desarrollado tomando como referencia diferentes fuentes de información que previamente abordaron temas similares al expuesto dentro de la presente investigación, en donde se encontró información variada, misma que fue procesada para la posterior construcción del estudio, en ella se procesaron un total de 27 estudios, distribuidos de la siguiente manera: 19 artículos científicos (Latindex, Scielo, Scopus), 2 libros, 5 proyectos de investigación y 1 informe que abordaron distintas temáticas donde el núcleo central fue el clima organizacional dentro del sector de la salud.

El conocimiento del clima organizacional en las distintas entidades de salud suministra retroinformación en relación a los procesos que determinan los comportamientos institucionales, permitiendo también introducir cambios planificados tanto en las conductas y actitudes de los miembros implicados, como en la estructura organizacional o en uno o más de los subsistemas que la conforman. En tal virtud, la importancia de esta información se enmarca en la comprobación de que el clima organizacional influye de forma directa en el comportamiento de cada uno de los miembros de la organización, mediante percepciones estabilizadas que filtran la realidad y condicionan los niveles de motivación laboral y el rendimiento de los distintos profesionales involucrados en el sector de la salud.

De forma general, se identifica que el ambiente de trabajo juega un papel indispensable en el comportamiento de los trabajadores y superiores, dado que engloba el conjunto de objetivos, valores, creencias, fines y reglas de funcionamiento a nivel individual, grupal y organizacional. Por lo tanto, el hecho de realizar estudios sobre esta variable permite determinar la forma en que los individuos perciben su trabajo, el desempeño, la productividad y las relaciones interpersonales con jefes y compañeros, factores que a su vez permiten identificar oportunidades de mejoras y puntos fuertes con la finalidad de cerrar las brechas de satisfacción entre la institución, sus empleados y la sociedad en general. 


\section{Referencias Bibliográficas}

Artaza, O., Barría, M. S., Fuenzalida, A., Nuñez, K., Quintana, A., Vargas, I., ... Vidales, A. (2016). Modelo de gestión de establecimientos hospitalarios ( $\mathrm{N}^{\circ}$ 9). Recuperado de http://www.bibliotecaminsal.cl/wp/ wp-content/uploads/2016/03/9.pdf.

Berberoglu, A. (2018). Impact of organizational climate on organizational commitment and perceived organizational performance: Empirical evidence from public hospitals. BMC Health Services Research, 18(1), 1-9. https://doi.org/https://doi.org/10.1186/s12913-018-3149-z

Bernal González, I., Pedraza Melo, N. A., \& Sánchez Limón, M. L. (2015). El clima organizacional y su relación con la calidad de los servicios públicos de salud: diseño de un modelo teórico. Estudios Gerenciales, 31(134), 8-19. Retrieved from http://dx.doi.org/10.1016/j.estger.2014.08.003

Bonilla Jurado, D., Macero Méndez, R., \& Mora Zambrano, E. (2018). La importancia de la capacitación en el rendimiento del personal administrativo de la Universidad Técnica de Ambato. Conrado, 14(63), 268-273. Epub 08 de junio de 2018. Recuperado en 08 de enero de 2020, de http://scielo.sld.cu/scielo.php?script=sci_arttext\&pid=S1990-86442018000300268\&lng=es\&tlng=es.

Bronkhorst, B., Tummers, L., Steijn, B., \& Vijverberg, D. (2015). Organizational climate and employee mental health outcomes: A systematic review of studies in health care organizations. Health Care Management Review, 40(3), 254-271.

Contreras, V., Reynaldos, K., \& Cardona, L. C. (2015). Clima, ambiente y satisfacción laboral: un desafío para la enfermería. Revista Cubana de Enfermería, 31(1), 58-69.

De La Cruz, E., \& Huaman Ruíz, Á. A. (2016). Clima organizacional y desempeño laboral en el personal del programa nacional cuna más en la provincia de Huancavelica - 2015. Universidad Nacional de Huancavelica.

Dos Santos Alves, D. F., \& Guirardello, E. de B. (2016). Nursing work environment, patient safety and quality of care in pediatric hospital. Revista Gaúcha de Enfermagem, 37(2), 1-7. https://doi.org/http://dx.doi. org/10.1590/1983- 1447.2016.02.58817

Forrellat Barrios, M. (2014). Calidad en los servicios de salud: un reto ineludible. Revista Cubana Hematología, Inmunología y Hemoterapia, 30(2), 179-183.

Gan, F., \& Berbel, G. (2007). Manual de Recursos Humanos. Barcelona, España: Editorial UOC.

García Solarte, M. (2009). Clima organizacional y su diagnóstico: Una aproximación conceptual. Cuadernos de Administración, (42), 43-61.

Githinji, N., \& Gachunga, H. (2017). Influence of organizational climate on employee performance in state corpo- 
rations in Kenya: A case of Kenya Industrial Estates limited. The Strategic Journal of Business \& Change Management, 4(2), 376-395.

Gruesso Hinestroza, M. P. (2016). Organizaciones saludables y procesos organizacionales e individuales. Comprensión y retos. Bogotá, Colombia: Editorial Universidad del Rosario.

Guevara, X. (2018). Clima organizacional y nivel de satisfacción en la Unidad Educativa Particular La Dolorosa (tesis de posgrado). Universidad Andina Simón Bolívar, Quito.

Hassan Kassem, A., \& Gaber, H. (2015). Organizational climate and its impact on nurse's job empowerment at Main Mansoura University Hospital and Urology and Nephrology Center. Journal of Nursing and Health Science, 4(2), 40-48. https://doi.org/10.9790/1959-0424048

Hernández, R. y Mendoza, C. (2018) Metodología de la investigación: las rutas cuantitativa, cualitativa y mixta. México D.F., México: McGrawHill

Iglesias Armenteros, A. L., \& Sánchez García, Z. T. (2015). Generalidades del clima organizacional. Medisur, 13(3), 455-457.

López Chanez, F. J., Casique Guerrero, A., \& Díaz Acevedo, N. B. (2014). Estudio de clima laboral en instituciones de Salud Pública. Congreso Internacional de Investigación En Ciencias Administrativas, (XV), 84-98.

Mohamadzadeh Nojehdehi, M., Ashgholi Farahani, M., Rafii, F., \& Bahrani, N. (2015). A comparison of organizational climate and nurses' intention to leave among excellence awarded hospitals and other hospitals in 2013. Iranian Red Crescent Medical Journal, 17(5), 1-6.

Montoya Agudelo, C. A., \& Boyero Saavedra, M. R. (2016). El recurso humano como elemento fundamental para la gestión de calidad y la competitividad organizacional. Revista Científica Visión de Futuro, 20(2), 1-20.

Munyewende, P. O., Rispel, L. C., \& Chirwa, T. (2014). Positive practice environments influence job satisfaction of primary health care clinic nursing managers in two South African provinces. Human Resources for Health, 12(1), 1-14. https://doi.org/10.1186/1478-4491-12-27

Pedraza Melo, N. A., \& Bernal González, I. (2018). El clima organizacional en el sector público y empresarial desde la percepción de su capital humano. Espacios, 39(13), 1-16.

Pérez Herrera, Y. (2014). Clima organizacional en el ámbito empresarial cubano (tesis de posgrado). Universidad de la Habana, La Habana.

Pinpin Choi, S., Cheung, K., \& Meiche Pang, S. (2012). Attributes of nursing work environment as predictors of registered nurses' job satisfaction and intention to leave. Journal of Nursing Management, 21(3), 1-11. https://doi.org/10.1111/j.1365-2834.2012.01415.x 
Ramírez Campos, Á. F., \& Domínguez Aguirre, L. R. (2012). El clima organizacional y el compromiso institucional en las IES de Puerto Vallarta. Investigación Administrativa, (109), 21-30.

Romero Veléz, E. M., Souto Anido, L., \& García Rondón, I. (2018). Procedimientos aplicados para el análisis del clima organizacional en el servicio público. Caso Ecuador. Observatorio de La Economía Latinoamericana, (Mayo 2018), 1-20.

Santana Del Salto, M. C. (2018). Comportamiento organizacional para el manejo de conflictos laborales en Instituciones de Salud Pública en la ciudad de Ambato (tesis de posgrado). Universidad Técnica de Ambato, Ambato.

Segredo Pérez, A. M., \& Reyes Miranda, D. (2015). Clima organizacional en salud pública. Consideraciones generales. Escuela Nacional de Salud Pública de Cuba, 1-8.

Yataco Tasayco, C. E. (2014). Clima organizacional y evaluación de la gestión institucional del colegio Túpac Amaru de la red 09 de la sede administrativa UGEL $N^{\circ} 01$ - del distrito de Villa María del Triunfo. Lima Sur - 2013 (tesis de posgrado). Universidad Nacional Mayor de San Marcos, Lima. 
